U.S. Department of Commerce, Bureau of Standards

RESEARCH PAPER RP620

Part of Bureau of Standards Journal of Research, Vol. 11, December 1933

\title{
COMPARISON OF NATURAL AGING OF PAPER WITH ACCELERATED AGING BY HEATING
}

\author{
By Royal H. Rasch ${ }^{1}$ and B. W. Scribner
}

\section{ABSTRACT}

Thirty-three papers ranging in fiber composition from 100 percent rag to 100 percent sulphite, including purified wood fiber papers, and book papers prepared from mixtures of sulphite and soda pulp, were tested after 4 years of normal aging for chemical purity and strength. The chemical properties of the papers showed little change under the tests applied. The folding endurance of the papers, however, underwent marked changes, several decreasing to less than half of the initial figure. The papers were placed in approximately the same order of stability by normal aging as they were by the accelerated aging tests ( 72 hours at $100^{\circ}$ C.) conducted 4 years previously. This constitutes additional evidence of the validity of the heat test as a means of estimating the relative aging quality of papers.

\section{CONTENTS}

Page

I. Introduction

II. Test methods_.

III. Results

IV. Conclusion

\section{INTRODUCTION}

In 1928 study was made at the Bureau of Standards of a series of 37 commercial papers with particular reference to chemical purity, strength, and relative permanence as estimated by an accelerated aging test. The portion of this study already published deals principally with the physical properties of the papers and the extent of their alteration under accelerated aging: ${ }^{2}$ The papers included in the study were, for the most part, writing papers ranging in fiber furnish from 100 percent sulphite to 100 percent rag. Four papers prepared from wood fibers of high chemical purity, and 5 book papers made from mixtures of sulphite and soda pulp were included.

Retests have been made recently of 33 of the papers for strength, alpha cellulose content, copper number, and acidity, after having been stored for 4 years. It is the purpose of this article to indicate briefly the changes, particularly in folding endurance, which have occurred during this relatively short period of normal aging in relation to the initial characteristics of the papers, and to the results of the accelerated aging tests made 4 years previously.

The papers were stored in a tightly closed bookcase in an office room free from abnormal atmospheric conditions, and each test sample was kept enclosed in a separate envelope, in order to minimize

1 Research Associate, at the Bureau of Standards, for the Brown Co., Berlin, N.H.

2 R. H. Rasch, A Study of Purified Wood Fibers as a Paper-Making Material, B.S. Jour. Research, vol. 3 (RP107), p. 469, 1929. 
any possible effect of external deteriorative influences. The data on alpha cellulose content, copper number, and acidity contained in this article were, for the most part, not included in the previous article which this supplements.

\section{TEST METHODS}

In testing the papers, the official methods of the Technical Association of the Pulp and Paper Industry were used for the determination of weight, bursting strength, thickness, folding endurance, breaking load, tearing strength, ash, resin, and glue. ${ }^{3}$ The procedure for the determination of alpha cellulose content and copper number have been described in a previous publication. ${ }^{4}$ Determinations of titratable acidity were made by the Kohler-Hall method, but the acidity is expressed as percent sulphuric anhydride rather than as acid number. ${ }^{5}$ The accelerated aging or stability test used consists in exposing the paper specimen under test to a current of air at $100^{\circ} \mathrm{C}$. for 72 hours and then determining to what extent the paper has been altered in its properties. This test has been described in previous publications. ${ }^{6}$

\section{RESULTS}

The test results are given in table 1 , which also contains descriptions of the papers and the more important original test data. For additional information on the characteristics of the papers, the reader is referred to the previous publication. ${ }^{7}$ In a few cases complete tests were not made. Four years of normal aging resulted in decreases in the folding endurance of most of the papers, which were quite large for many of them, some decrease in tearing strength and bursting strength for the least stable papers, and no appreciable change in the breaking load of any of the papers. The acidity increased slightly in most cases, the greatest changes being recorded for the sulphite bond and sulphite-soda book papers. The copper numbers of the papers were unaltered within the limits of experimental error. The alpha cellulose contents of the sulphite bond papers and two of the sulphite-rag papers underwent slight decreases. Similar changes in the various properties were obtained with the 72-hour heat treatment at $100^{\circ} \mathrm{C}$., except that this treatment had a uniformly greater deteriorative effect. It caused decreases in alpha cellulose content and increases in copper number, which in most instances did not take place under normal aging for 4 years.

The results of normal aging bear out the heat tests in showing that the folding endurance is the most sensitive of the tests here discussed for indicating the relative stabilities of the papers.

\footnotetext{
3 Paper Testing Methods, prepared by the Committee on Paper Testing of the Technical Association of the Pulp and Paper Industry, Lockwood Trade Jour. Co., New York, 1929.

Burton, J. O., and Rasch, R. H., The Determination of the Alpha Cellulose Content and Copper Number of Paper, B.S.Jour. Research, vol. 6 (RP295), p. 603, 1931.

$s$ Kohler, S., and Hall, G., Acidity in Paper, Paper Industry, vol. 7, no. 7, p. 1, 1925.

6 Rasch, R. H., Accelerated Aging Test for Paper, B.S.Jour. Research, vol. 7 (RP352), p. 465, 1931. See also footnote 2, p. 727 .

7 See footnote 2, p. 727 .
} 


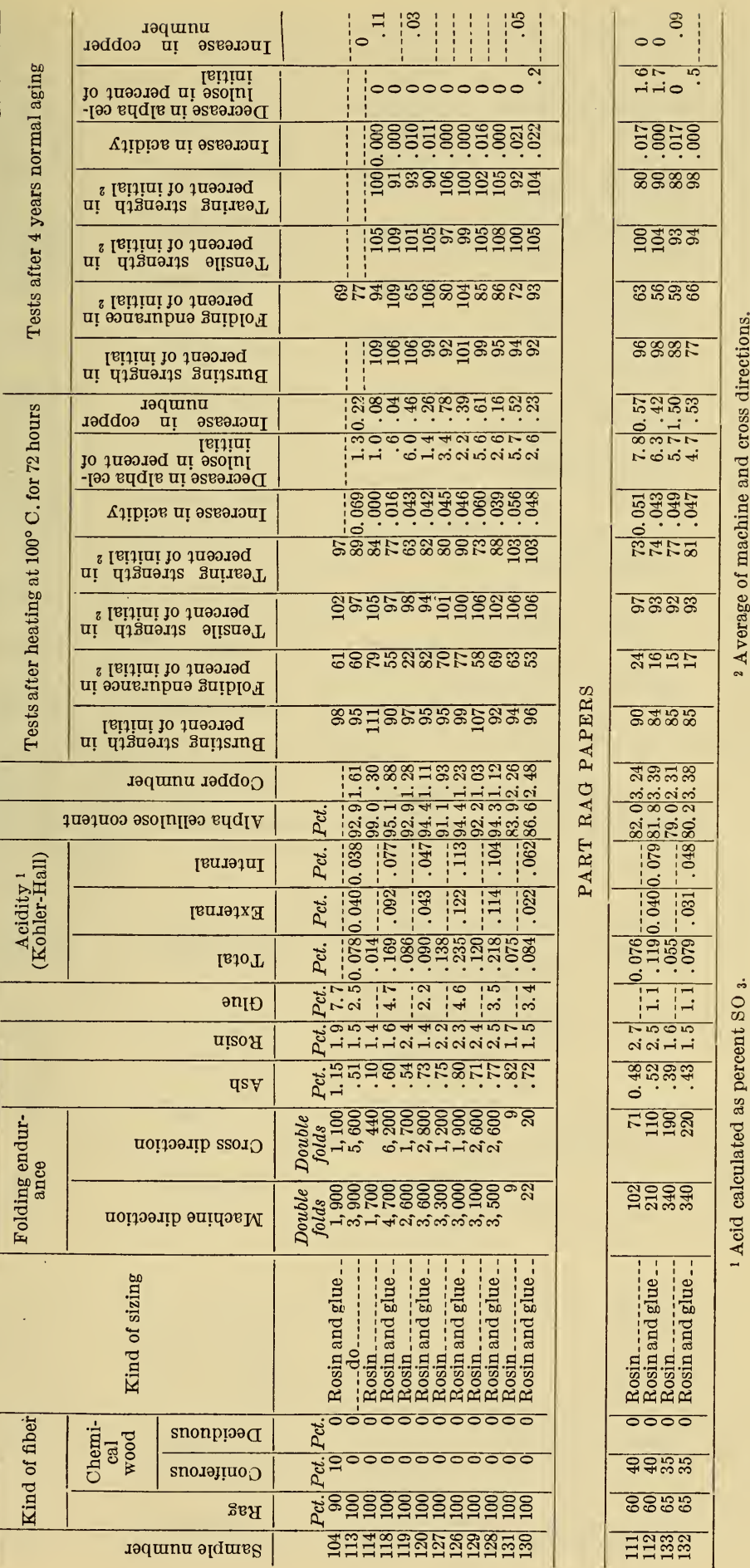




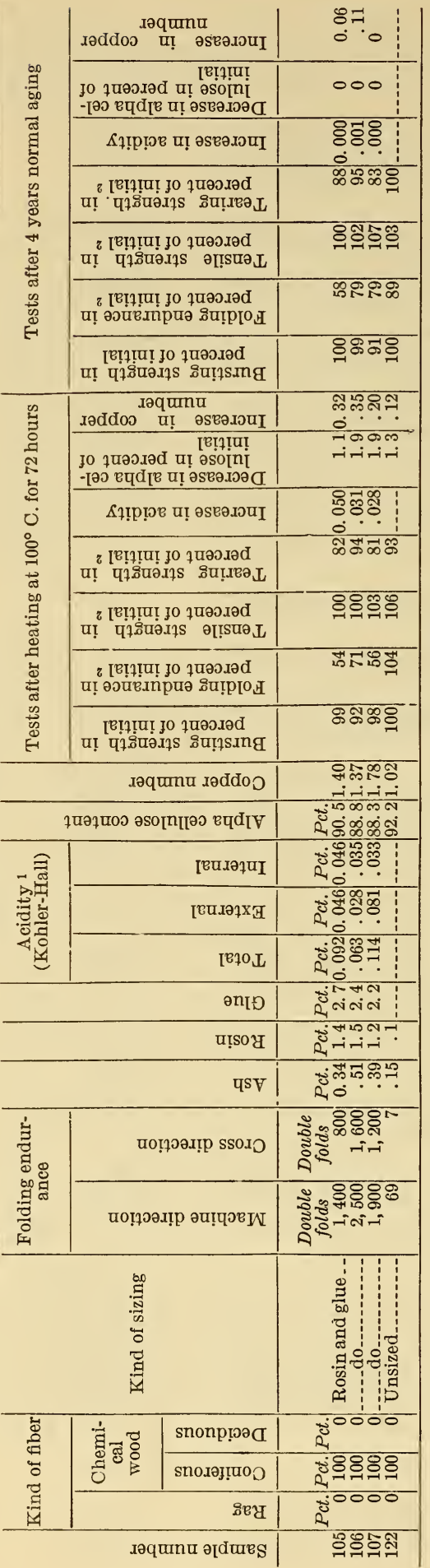

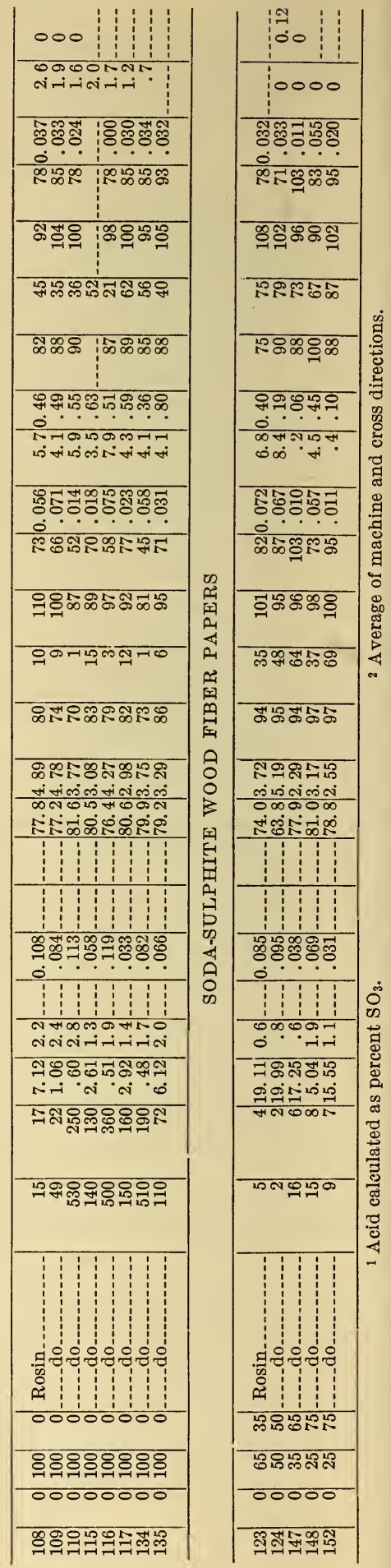


Figure 1 shows the relation between the percentage retention of folding endurance after 4 years normal aging and the percentage retention of folding endurance after heating at $100^{\circ} \mathrm{C}$. for 72 hours. Although some of the points, particularly in the range of higher stability, are rather widely scattered it is evident that in general the folding endurance of those papers least stable under the heat test decreased at a faster rate during the 4 years of normal aging than that of the papers which were more stable toward heat. Similarly, the papers most resistant toward heat showed the least change in folding endurance under normal aging.

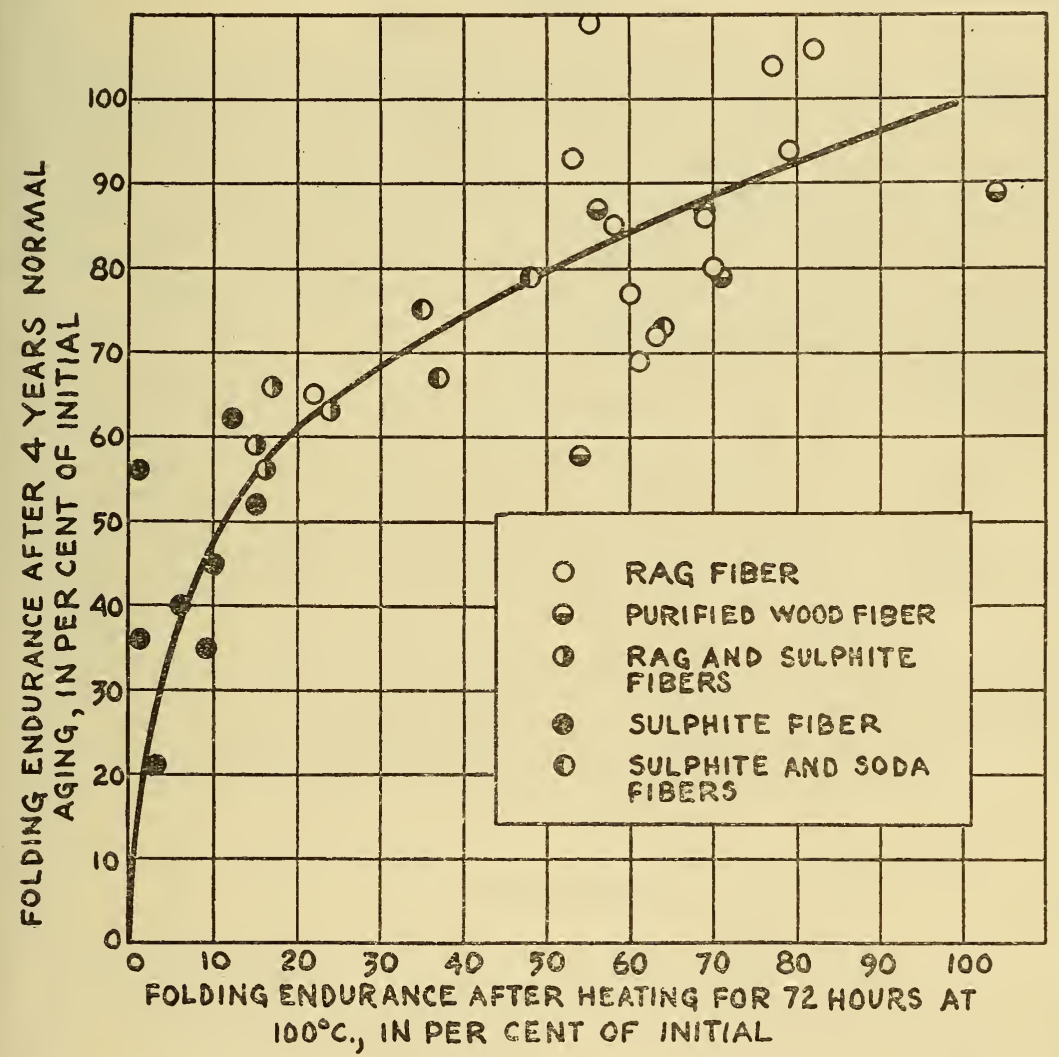

FIgURE 1.-Comparison of retention of folding endurance after normal and accelerated aging.

In general, the papers with the highest chemical purity were the most resistant to deterioration either by heat or normal aging, while those with the least desirable initial properties underwent more rapid deterioration. It will be noted that the most significant relation of the initial properties of these papers to their weakening during storage, and likewise under the heat test, was the purity of the fibers as measured by alpha cellulose content and copper number. An exception appears in the case of the soda-sulphite book papers, which, despite comparatively low alpha cellulose contents and high copper numbers, have fold retention values which are intermediate between those of 
the best and poorest papers. It should be noted, however, that these values are probably somewhat inaccurate because of the extremely low initial folding endurance values. However, it is noteworthy that the same exception occurred under the heat test. This was ascribed ${ }^{8}$ to the several differences in the components of this type of paper and writing paper.

It seems desirable to mention that as these papers were 4 years old, they should not be regarded as necessarily representative of the average quality of such papers at present because improvement in properties that affect permanence of papers has been the general rule during this period.

\section{CONCLUSION}

Despite the several discrepancies within the groups of similar papers, which are to be expected with such a short period of natural aging, the results of these retests of stored papers constitute further evidence of the validity of the heat test for approximate estimation of the relative aging qualities of papers, and also give further evidence of the close relation of fiber purity to these qualities. The storage of the papers is being continued for further retests of them.

Washington, September 16, 1933.

${ }^{8}$ See footnote 2, p. 727. 